\title{
Eogitare
Enfermagem
}

\section{PROGRAMA DE EDUCAÇÃO PARA A AUTOGESTÃO EM ADO- LESCENTES COM SPINA BÍFIDA: O QUE DIZEM OS JOVENS E SEUS CUIDADORES}

Maria Isabel Dias da Costa Malheiro1, Maria Filomena Gaspar², Luísa Barros³

\section{RESUMO}

Objetivo: avaliar um programa e os seus efeitos nas competências de autogestão, na perspetiva dos jovens e de seus pais/cuidadores.

Método: entrevistas em grupo focal, com os 51 jovens com Spina Bifida (10-18 anos) que realizaram o programa e 30 pais/cuidadores.

Resultados: revelam melhoria no comportamento de autogestão dos jovens, mais responsáveis, proactivos, confiantes, competentes na resolução de problemas, e autónomos. Quanto ao programa, destacam as estratégias psicoeducativas utilizadas, como a técnica de resolução de problemas, o roleplaying, os vídeos, o regime de tutoria entre pares e a modelagem (Lay led's).

Conclusão: o programa obteve resultados positivos na capacitação dos jovens para a autonomia. As mudanças verificadas no jovem ao assumir o papel de tutor permitem destacar a eficácia desta estratégia e o seu potencial na criança com condição crónica.

DESCRITORES: Programa; Capacitação; Adolescentes; Condição Crónica; Autogestão.

COMO REFERENCIAR ESTE ARTIGO:

Malheiro MIC, Gaspar MF, Barros L. Programa de educação para a autogestão em adolescentes com spina bífida: o que dizem os jovens e seus cuidadores. Cogitare enferm. [Internet]. 2019 [acesso em "colocar data de acesso, dia, mês abreviado e ano"]; 24. Disponível em: http://dx.doi.org/10.5380/ce.v24i0.62222.

${ }^{1}$ Enfermeira. Doutora em Enfermagem. Docente da Escola Superior de Enfermagem de Lisboa. UI\&DE. Lisboa, Portugal (i) ${ }^{2}$ Enfermeira. Doutora em Gestão em Saúde. Docente da Escola Superior de Enfermagem de Lisboa, UI\&DE. Lisboa, Portugal. (1)

3Psicóloga. Doutora em Psicologia. Docente na Faculdade de Psicologia de Lisboa. Lisboa, Portugal. (1) 


\title{
EDUCATIONAL PROGRAM FOR SELF-MANAGEMENT ON ADOLES- CENTS WITH SPINA BIFIDA: WHAT DO YOUTHS AND THEIR CARE- GIVERS HAVE TO SAY?
}

\begin{abstract}
Objective: To evaluate an educational program and its effects on self-management skills, from the perspective of youths and their parents/caregivers.

Method: The present study conducted interviews in focus groups with 51 youths with spina bifida (10-18 years old) who participated in the program and 30 parents/caregivers.

Results: Improvements were observed in the youths' self-management skills, who displayed greater responsibility, proactivity, confidence, problem-solving skills and autonomy. In terms of the psychoeducational strategies used in the program, emphasis goes to the problem-solving technique, role-playing, videos, peer mentorship, and modeling (lay led model).

Conclusion: The results of the program showed that the youths built skills that fostered their autonomy. The changes observed in the youths on taking on the role of mentors point to the effectiveness of this strategy and its potential for children with chronic conditions.
\end{abstract}

DESCRIPTORS: Program; Education; Adolescents; Chronic Illness; Self-Management.

\section{PROGRAMA EDUCATIVO DE AUTOGESTIÓN PARA ADOLESCENTES CON ESPINA BÍFIDA: QUÉ DICEN LOS JÓVENES Y CUIDADORES}

\section{RESUMEN}

Objetivo: Evaluar un programa y sus efectos en las competencias de autogestión, conforme la visión de los jóvenes y de sus padres/cuidadores.

Método: Entrevistas en grupo focal, con los 51 jóvenes con espina bífida (10-18 años) que realizaron el programa y 30 padres/cuidadores.

Resultados: Expresan mejora conductual de la autogestión de los jóvenes, más responsables, proactivos, confiados, competentes para resolver problema, autónomos. Respecto del programa, destacan las estrategias psicoeducativas utilizadas, como la técnica de resolución de problemas, el roleplaying, los vídeos, el régimen de tutoría entre pares y el modelado (Lay led's).

Conclusión: El programa tuvo resultados positivos en la capacitación de los jóvenes para su autonomía. Los cambios verificados en el joven al asumir el rol de tutor permiten destacar la eficacia de esta estrategia y su potencial en el niño con enfermedad crónica.

DESCRIPTORES: Capacitación; Adolescentes; Enfermedad Crónica; Autogestión. 
Os avançostecnológicos, assim como ocrescente desenvolvimento dos conhecimentos na área da saúde infantil, contribuíram, por um lado, para um aumento da esperança de vida de crianças cuja sobrevivência era pouco provável num passado recente, por outro, para que a taxa de morbilidade infantil tenha aumentado e um número significativo destas crianças apresente necessidades especiais. A Spina Bífida (SB), sob a forma de mielomeningocelo, surge como uma destas situações: uma malformação congénita caracterizada pelo incompleto encerramento do tubo neural durante a fase embrionária. Trata-se de uma condição complexa que provoca alterações do foro sensoriomotor (paralisia e analgesia dos membros inferiores), urológico ( $95 \%$ apresentam bexiga neurogénica, incontinência do esfíncter vesical) e intestinal (incontinência do esfíncter anal)(1). Estas crianças são diariamente confrontadas com tarefas de grande complexidade como a necessidade de realizar a autoalgaliação intermitente a cada 3 horas, treino intestinal e supervisão da pele (elevada incidência de úlceras de pressão e lesões cutâneas) ${ }^{(2)}$.

A taxa de sobrevivência nestas crianças até à idade adulta aumentou significativamente nas últimas décadas $95 \%{ }^{(3)}$. Um elevado número destes jovens atingem a idade adulta ainda muito dependentes dos seus pais/cuidadores na realização das Atividades de vida diária (AVD). A literatura descreve as crianças com SB com níveis inferiores de autonomia na tomada de decisão e na realização do autocuidado, sobretudo nas áreas da eliminação, higiene e transferências ${ }^{(4)}$. Uma elevada percentagem dos adultos com SB necessita de assistência da família no autocuidado ( $43 \%$ moderada e $24 \%$ mínima) e na gestão da condição(5).

Viver e conviver com uma deficiência requer que o jovem com SB seja capaz de gerir um manancial de necessidades especiais de forma autónoma e independente, situação que constitui um desafio não só para os indivíduos com SB e sua família, como também para os profissionais de saúde que os acompanham.

O desenvolvimento de competências para levar a cabo estas tarefas é fundamental para a manutenção da saúde do jovem e também para uma transição para a vida adulta o mais harmoniosa possível(2). Assim, a transição para a vida adulta constitui um desafio para os profissionais de saúde que seguem estas crianças, sendo o objetivo da intervenção do enfermeiro facilitar a transferência da responsabilização pela gestão dos cuidados especiais de saúde de forma progressiva, dos pais/cuidadores para os jovens ${ }^{(6)}$.

O desenvolvimento das competências de autogestão no jovem com condição crónica irá permitir que este aprenda a viver, trabalhar e participar ativamente na sociedade, com conhecimento de como gerir a sua condição e como prevenir a curto e longo prazo as complicações. Embora a manutenção da saúde física e a melhoria da funcionalidade motora sejam fatores importantes, os jovens com SB necessitam desenvolver competências de autogestão e independência para, mais tarde, "sobreviverem" no mundo dos adultos ${ }^{(7)}$. Tal obriga a uma mudança no foco de cuidados de saúde durante a infância e a adolescência, através do encorajamento e ajuda à responsabilização do próprio pela gestão da sua condição, preparando-se, deste modo, para assumir os papéis inerentes à idade adulta ${ }^{(8)}$.

A maioria dos adultos com SB tem potencialidade para ser totalmente autónomo e ter um estilo de vida muito perto do "normal". A criança, o adolescente ou o adulto devem estar bem equipados com competências, de forma a atingirem um grau de independência que lhes permita "sobreviver" num mundo competitivo, gerindo adequadamente a sua vida, muitas vezes complexa ${ }^{(9)}$. O processo deve iniciar-se na infância e focalizar-se na promoção da mestria nas tarefas fundamentais, como a aquisição da autonomia e a participação ativa na comunidade onde está inserido, alcançando assim uma melhoria da qualidade de vida e bem-estar ${ }^{(10)}$.

A elaboração de programas de intervenção educativa focalizados na funcionalidade, numa perspetiva desenvolvimentista, pode constituir a chave para minimizar a incapacidade e maximizar as capacidades de modo a que estes encontrem estratégias adaptativas que 
Ihes permitam um desenvolvimento harmonioso e o mais funcional e autónomo possível(9).

Para dar resposta a esta problemática tem-se dado ênfase a estratégias psicoeducativas, no sentido de facilitar o processo de adaptação à condição crónica. Entre os diversos programas de educação para a autogestão encontrados na literatura, destacase o modelo de intervenção desenvolvido por Kate Lorig Expert Patients Programme. Foram pioneiros na utilização de Lay leds no programa (modelo com a mesma condição crónica dos participantes e que é perito na autogestão da sua condição), programa cuja evidência científica comprovou ser eficaz em adultos e idosos com condição crónica ${ }^{(11)}$. Mais tarde foi adaptado à pediatria por Malheiro e implementado em adolescentes com Spina Bifida num estudo piloto, cujos resultados revelaram melhoria significativa nos níveis de funcionalidade ${ }^{(12)}$ e nas competências de autogestão (resolução de problemas relacionados com os cuidados especiais, responsabilidade pela gestão do regime terapêutico) ${ }^{(13)}$. Os autores referem as vantagens na utilização de estratégias psicoeducativas no programa, sendo que os adolescentes destacam as estratégias reforço da autoeficácia de Bandura como aquelas que demonstraram ser mais efetivas como a persuasão social (a partilha de experiências e problemas com pares em situação semelhante) e a modelagem (a utilização de modelos de referência, vídeos, simulação e a dramatização/roleplaying)(13).

Deste modo, e no sentido de validar a efetividade do programa, este foi implementado a mais 51 adolescentes com Spina Bífida e avaliados os seus efeitos no comportamento pelos adolescentes após a realização do programa e pelos seus pais/cuidadores após seis meses de convivência no domicílio.

\section{MÉTODO}

O presente estudo de caráter descritivo e exploratório constitui uma componente qualitativa de um largo estudo, quasi-experimental e longitudinal. Quanto ao desenho do estudo, os autores recomendam uma abordagem mista para enriquecer os resultados com informação contextual significativa e uma detalhada descrição da experiência ${ }^{(14)}$. Deste modo, no final da intervenção, foi feita uma avaliação do programa e seus efeitos pelos jovens (após a realização do programa) e pelos os pais/cuidadores no follow-up (após 6 meses de convivência com os jovens no domicílio).

O programa é composto por 7 sessões (1 ${ }^{\text {a }}$ Sessão: "Viver com Spina Bífida e a Importância da Autonomia", $2^{\mathrm{a}}$ Sessão: "Úlceras de Pressão - Prevenção e deteção

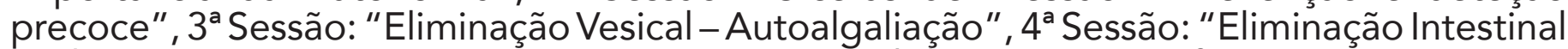
- Alimentação e Exercício Físico", $5^{\mathrm{a}}$ Sessão: "Mobilização \& Transferências", $6^{\mathrm{a}}$ Sessão: "Higiene Nestir \& Despir" e na $7^{\mathrm{a}}$ Sessão: "O Regresso a Casa" (estratégias de confronto face aos comportamentos de superproteção) e avaliação qualitativa do programa através de entrevistas de grupo focal.

O programa foi implementado em dois grupos distintos, constituídos por Grupo 1: raparigas (9h30 às $11 \mathrm{~h}$ ) e Grupo 2: rapazes (11 h às $12 \mathrm{~h} 30)$, durante 7 dias.

Todas as sessões seguiram um protocolo detalhado (manual de suporte) com as seguintes etapas: Breve introdução ao tema; Questionamento (identificar os conhecimentos dos participantes relativamente à temática); Brainstorming de Problemas (listar os problemas identificados pelos participantes relativamente ao tema da sessão); Técnica de Resolução de Problemas, utilizar alguns dos problemas anteriormente identificados (os mais frequentes) e questionar o grupo quanto às possíveis formas de resolução (listar soluções) e discutir as vantagens e desvantagens da resolução proposta; Roleplaying: simulação de dois problemas (identificados num estudo exploratório Matrix Assessment); Breve lição (apresentação sumária em slides/vídeo de alguns pontos-chave relacionados com o tema abordado na sessão); A elaboração do plano de ação (no final de cada sessão, os participantes comprometeram-se a mudar, pelo menos, um comportamento relacionado com o tema da sessão e registá-lo no seu livro individual). 
No decorrer do programa foi implementado o regime de tutoria com as seguintes orientações:

- Os tutores foram selecionados de entre os participantes pela idade (metade do grupo era tutor da restante metade). Supervisionam diariamente os registos do tutorando no livro individual e avaliaram os planos de ação elaborados no final de cada sessão (havendo um incentivo, com um "prémio", ao seu cumprimento).

As sessões foram realizadas pela mesma enfermeira e Lay Led (feminino no grupo das raparigas e masculino no grupo dos rapazes), decorreram sem incidentes, os jovens aderiram com bastante entusiasmo demonstrando uma participação ativa através do questionamento, intervenção, participação no roleplaying e elaboração dos planos de ação.

Participaram neste estudo cinquenta e um jovens $(n=51)$ com SB, que realizaram o programa na totalidade, e trinta pais/cuidadores $(n=30)$. Foram cumpridos todos os procedimentos éticos exigidos (declaração de Helsínquia), com o pedido de consentimento informado aos pais e assentimento aos jovens para a sua colaboração neste estudo.

O presente estudo foi aprovado pela Comissão Científica da Universidade de Lisboa e pela Comissão de Ética da Associação de Spina Bifida e Hidrocefalia de Portugal.

\section{RESULTADOS}

Relativamente à caraterização dos participantes, como podemos observar na Tabela 1, a média da idade dos participantes foi de 13.3, sendo que 24 (47\%) são do sexo masculino e 27 (52.9\%) feminino.

Tabela 1 - Caracterização dos participantes $(n=51)$

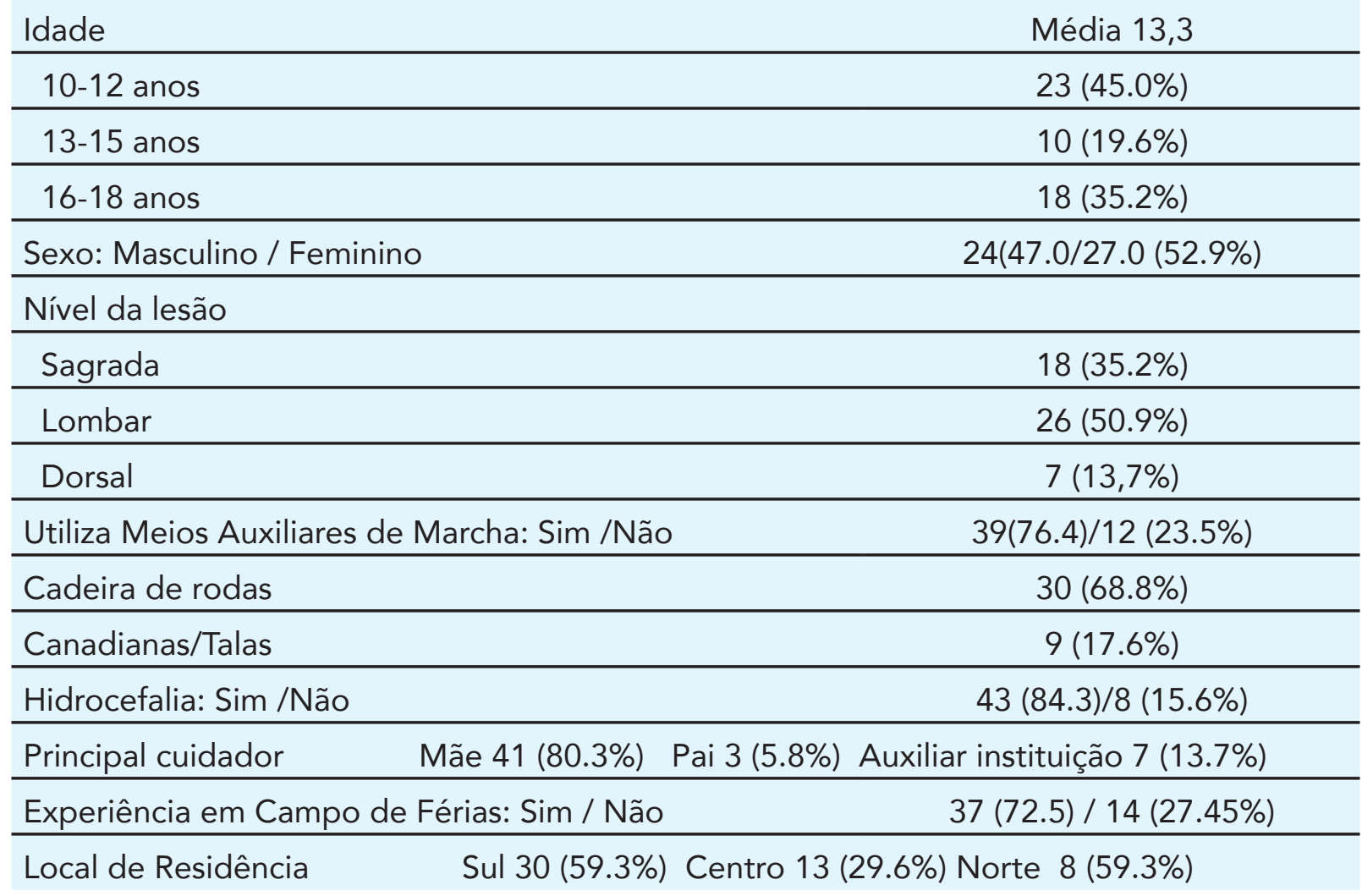


Como técnica de recolha de dados, optou-se por utilizar o Grupo Focal, uma técnica que vem sendo muito utilizada pelos investigadores na área das ciências sociais. Gibbs define como uma discussão cuidadosamente preparada, que tem como objetivo obter dados sobre as perceções do grupo acerca de determinada área de interesse ${ }^{(15)}$. Acrescenta ainda que esta discussão promove a interação entre os entrevistados, e, quando associada a uma atitude provocatória do entrevistador, origina um ambiente propício de espontaneidade para desencadear atitudes e emoções que dificilmente seriam verbalizados noutras situações ${ }^{(13)}$.

Foram realizadas 8 entrevistas de grupo focal a 51 jovens após a realização do programa, e 4 grupos focais a 30 pais/cuidadores seis meses após a realização do programa.

Para a análise dos dados, foi realizada a análise de conteúdo temática categorial segundo Bardin, com algumas particularidades relacionadas com a técnica de colheita de dados Grupo Focal ${ }^{(13)}$.

Procurou-se assegurar o rigor científico encontrando consistência interna nesta fase de "codificação", através da concordância entre a interpretação dos dados da investigadora e a dos juízes. A grelha de análise que podemos observar na Tabela 2 foi construída de um modo indutivo e em concordância com os respetivos juízes e que serviu de suporte ao modelo de análise global. Tal como efetuado no estudo piloto, sempre que os resultados o justifiquem, em termos de discrepância e/ou divergência, será feita uma análise entre grupos (Jovens e Pais/Cuidadores) ${ }^{(13)}$.

Tabela 2 - Grelha de análise

\begin{tabular}{|c|c|c|c|}
\hline DIMENSÃO & CATEGORIA & SUBCATEGORIAS & INDICADORES \\
\hline \multirow{11}{*}{ AVALIAÇÃO } & \multirow{9}{*}{ POSITIVA } & \multirow{3}{*}{ Comportamento do Jovem } & Responsabilidade \\
\hline & & & Proatividade \\
\hline & & & Atividade Física \\
\hline & & \multirow{3}{*}{ Perceção do Self } & Autoeficácia \\
\hline & & & Autoestima \\
\hline & & & Autoconfiança \\
\hline & & \multirow{3}{*}{ Competências } & Resolução de problemas \\
\hline & & & Autonomia AVD's \\
\hline & & & Interação social \\
\hline & \multirow{2}{*}{ NEGATIVA } & \multirow{2}{*}{ Comportamento do Jovem } & Regressão \\
\hline & & & Dependência \\
\hline
\end{tabular}

Procurar saber qual a opinião dos jovens e seus pais/cuidadores acerca do efeito do programa no comportamento dos jovens, pressupôs uma abordagem focalizada na avaliação dos resultados desta experiência e as razões que apontam para fundamentar essa opinião. $\mathrm{Na}$ análise dos dados obtidos, nesta dimensão, emergem duas categorias: avaliação positiva (Tabela 3) e avaliação negativa. 
Tabela 3 - Avaliação Positiva do Efeito do Programa "Campo"

\begin{tabular}{|c|c|c|c|c|}
\hline CATEGORIA & SUBCATEGORIAS & INDICADORES & JOVENS & PAIS/CUI \\
\hline \multirow{9}{*}{ POSITIVA } & \multirow{3}{*}{ Comportamento do Jovem } & Responsabilidade & 48 & 45 \\
\hline & & Proatividade & 8 & 28 \\
\hline & & Disciplina & 3 & 6 \\
\hline & \multirow{3}{*}{ Perceção do Self } & Autoestima & 14 & 37 \\
\hline & & Autoconfiança & 16 & 16 \\
\hline & & Autoeficácia & 3 & 19 \\
\hline & \multirow{3}{*}{ Competências } & Independência AVD's & 39 & 65 \\
\hline & & Resolução de problemas & 69 & 28 \\
\hline & & Interação social & 55 & 22 \\
\hline
\end{tabular}

Quando apontam as principais razões para justificar a avaliação positiva, os sujeitos de ambos os grupos referem-se às mudanças do comportamentonos jovens, à perceção do Self e, ainda, às competências que desenvolveram durante este processo.

Relativamente às mudanças comportamentais, ambos os grupos destacam de um modo bastante evidente um comportamento do jovem mais responsável no cumprimento das tarefas relacionadas com as suas necessidades especiais de saúde.

"tinha uma pessoa à minha responsabilidade e isso obrigou-me a pensar que tenho que ser responsável." (J)"ela veio mais responsável, veio ...mais crescida... tem mais responsabilidade ... cumpre os horários" $(P)$

Foi também referido, pelo grupo de pais, uma maior proatividade e autossufiência dos jovens relativamente ao seu autocuidado.

"...pede menos ajuda e só chama quando não consegue mesmo!" (P).

Relativamente à perceção do self, ambos os grupos referiram um aumento da autoeficácia: "aprendi a ser independente e a fazer ... a minha algaliação, e não preciso mais da minha mãe "(J), "Mais confiante a fazer as coisas, excelente!" (P). O aumento da autoestima "sentirmo-nos úteis e ajudar os miúdos que estão a passar aquilo que nós já passámos" (J) também evidenciado pelos pais "vem muito positiva e feliz!" (P).

Quanto às competências que referem ter adquirido, destacam a resolução de problemas e que justificam através das repercussões positivas no seu dia-a-dia: "...foi muito importante para estarmos preparados para os problemas que possam acontecer... a forma como os outros resolvem os problemas pode-nos ajudar no futuro"(J). Essas repercussões positivas também se refletem no discurso dos pais "...está mais desenrascada...resolve as coisas sem me dar satisfações"(P).

Referem maior independência na realização das atividades de vida diária: "(...) fiquei mais independente em tudo, fiz a minha mala, tratei de tudo o que era meu... foi importante." (J) "já não precisa de ninguém para o ajudar a algaliar na escola e em casa! Uma maravilha, já faz tudo sozinho na escola e em casa" (P).

Destacam ainda uma melhoria nas competências sociais pelas oportunidades de interação social que esta experiência lhes proporcionou:

"fiz amigos novos e que são para a vida (...)" "conheci o meu namorado!" (J) "arranjou um grupo de amigos e isso é muito importante, agora tenho que a levar a sair com os amigos 
e acho que tem namorado (riu-se)" $(P)$.

Como aspetos negativos relacionados com esta experiência, alguns pais referem uma regressão nos comportamentos já adquiridos e demonstrados no período pós campo, no entanto, ressalvam que esta regressão não é total. No entanto, alguns pais reconhecem que deveriam dar continuidade a estas aprendizagens em casa e que não o fazem por uma questão de comodidade, ser mais fácil e rápido fazer por eles.

Quanto à avaliação do Programa de Educação para a Autogestão, os jovens referemse a aspetos positivos e negativos relacionados com os temas das sessões e as estratégias psicoeducativas utilizadas (Tabela 4).

Tabela 4 - Grelha Análise: Avaliação do Programa

\begin{tabular}{|c|c|c|c|}
\hline DIMENSÃO & CATEGORIA & SUBCATEGORIAS & INDICADORES \\
\hline \multirow{14}{*}{$\begin{array}{l}\text { AVALIAÇÃO } \\
\text { PROGRAMA }\end{array}$} & \multirow{11}{*}{ POSITIVA } & \multirow{6}{*}{$\begin{array}{l}\text { Temática das } \\
\text { Sessões }\end{array}$} & Condição Clínica/SB/LM \\
\hline & & & Prevenção Terciária \\
\hline & & & Transferências \\
\hline & & & Autoalgaliação \\
\hline & & & Higiene, Vestir e Despir \\
\hline & & & Importância da Autonomia \\
\hline & & \multirow{5}{*}{$\begin{array}{l}\text { Estratégias } \\
\text { Psicopedagógicas }\end{array}$} & Role playing/simulação \\
\hline & & & Lay Leds/monitores com SB \\
\hline & & & Modelagem \\
\hline & & & Resolução de problemas \\
\hline & & & Regime Tutoria \\
\hline & \multirow{3}{*}{ NEGATIVA } & $\begin{array}{l}\text { Estratégias } \\
\text { Psicopedagógicas }\end{array}$ & Regime de Tutoria \\
\hline & & \multirow{2}{*}{$\begin{array}{l}\text { Temática das } \\
\text { Sessões }\end{array}$} & Não adequados às necessidades \\
\hline & & & Pouco Interessantes \\
\hline
\end{tabular}

Quanto à avaliação positiva que os jovens fazem relativamente ao programa, destacam alguns dos temas abordados e justificam a sua opinião fazendo referência à aprendizagem que lhes proporcionou e ainda as estratégias psicoeducativas utilizadas, como podemos observar pela Tabela 5.

Tabela 5 - Categoria: Avaliação Positiva do Programa (continua)

\begin{tabular}{llll} 
CATEGORIA & SUBCATEGORIAS & INDICADORES & JOVENS \\
\hline \multirow{2}{*}{ POSITIVA } & \multirow{2}{*}{ Temática das Sessões } & Transferências & 50 \\
\cline { 3 - 4 } & & Condição Clínica/SB/LM & 40 \\
\hline
\end{tabular}




\begin{tabular}{lll} 
& \multicolumn{1}{l}{ Importância da Autonomia } & 26 \\
\cline { 2 - 3 } & Autoalgaliação & 21 \\
\cline { 2 - 3 } & Prevenção Terciária & 27 \\
\cline { 2 - 3 } & Higiene, Vestir e Despir & 26 \\
\hline \multirow{3}{*}{ Estratégias Psicopedagógicas } & Resolução de problemas & 78 \\
\cline { 2 - 3 } & Role playing/simulação & 57 \\
\cline { 2 - 3 } & Regime Tutoria & 54 \\
\cline { 2 - 3 } & Modelagem & 40 \\
\cline { 2 - 3 } & Lay Leds/monitores com SB & 40
\end{tabular}

Relativamente à subcategoria Temática das Sessões, os jovens destacam algumas áreas que consideraram mais importantes, tais como: as transferências da cadeira de rodas; a sessão sobre a condição SB: "conhecer melhor a nossa doença", "aprendi muitas...coisas sobre spina bífida"; o procedimento da autoalgaliação:"Aprendi a fazer a algaliação" "elas sabem como ensinar pois também tiveram que aprender e isso é bom" (J), e ainda a importância da autonomia nas AVD's: "Estas sessões ajudaram-me a perceber a importância de sermos independentes e fazermos as coisas por nós próprios, não vamos andar sempre com a mãe atrás" (J).

Relativamente às estratégias psicoeducativas utilizadas no programa, os jovens destacam de um modo evidente a estratégia de resolução de problemas: "perceber que todos temos os mesmos problemas" o desafio para as resolvemos...isso ajuda (J), seguindose o roleplaying/simulação e os vídeos "mais filmes ... com os monitores a mostrar como se deve fazer" "(J), "...fazer de pai e de mãe e perceber o que eles sofrem a tentar que nós façamos as coisas bem, foi difícil"(J)....fazer de Enfermeiro e perceber o difícil que é explicar-nos"(J).

O regime de tutoria entre pares emerge como uma estratégia que lhes proporcionou uma mais-valia em termos de aprendizagem e aquisição da sua autonomia: "aprendi muito" "para ensinar a minha criança, tive que aprender e saber fazer tudo muito bem" (J) "grande responsabilidade que nos prepara para ser monitores"(J).

A utilização dos Lay leds também foi valorizada pelos jovens, não só pela oportunidade de aprendizagem com um adulto portador de Spina Bífida com mais experiência, mas sobretudo associada à hipótese de um dia também poderem vir a ser monitores no campo: "É muito importante ter os monitores com Spina Bífida nestes programas" "eles sabem como fazer as coisas pois já as tiveram que aprender" "O meu sonho é ser monitora" (J).

\section{DISCUSSÃO}

O presente estudo procurou avaliar os efeitos de um programa de educação para a promoção da autogestão em adolescentes com SB em contexto de Campo de Treino, na perspetiva dos jovens e dos seus pais/cuidadores. A avaliação do programa feita pelos participantes e pelos pais/cuidadores foi claramente positiva. São evidentes os ganhos relacionados com as competências na realização das AVD's e de autogestão da condição e que se mantêm seis meses após a realização do programa. Os pais destacam não só os ganhos em termos de autonomia relacionados com o desempenho nas tarefas diárias, mas, essencialmente, na atitude mais proativa e responsável no cumprimento dos cuidados especiais de saúde. 
Surpreendentemente, os participantes revelaram escassos conhecimentos acerca da sua condição, demonstrando muito interesse em a conhecer melhor o que é a SB, quais as implicações e como se manifestam. Apesar de $82 \%$ dos jovens terem hidrocefalia (associada ao Síndrome Arnold Chiari) e válvula ventrículo-peritoneal, nenhum soube explicar o que significava. O especial interesse manifestado pela sessão da higiene - com deteção precoce de úlceras de pressão - pode estar relacionado com a preocupação destes jovens relativamente à elevada incidência de úlceras de pressão(16).

Quanto às estratégias psicoeducativas utilizadas, os jovens destacam as mais dinâmicas e interativas, referindo serem as que mais os divertiam e que lhes permitiram treinar competências de autogestão da condição ${ }^{(13)}$. A aprendizagem através da resolução de problemas permitiu-lhes treinar esta competência nas diversas fases: identificação do problema, conhecer e refletir sobre as várias hipóteses de o resolver (em grupo), avaliar os prós e os contras relativamente às consequências de cada opção e tomar uma decisão que vise a sua solução. Esta metodologia permite que o jovem adquira confiança no seu processo de autonomia ${ }^{(17)}$. Resultado já encontrado no estudo piloto e pode ser comprovado pelos resultados obtidos no nosso estudo, em que os jovens referem sentir-se mais confiantes, proativos e competentes na resolução dos seus problemas, o que é confirmado pelos seus pais/cuidadores após seis meses de convivência com eles no domicílio.

O roleplaying/dramatização de situações problema mais uma vez constituiu um importante momento de aprendizagem para o grupo. Assumir o papel de pais ou profissionais de saúde e sentir na primeira pessoa as dificuldades inerentes a uma intervenção que visa a mudança de comportamentos inadequados e nocivos para a saúde, foi considerado um desafio para eles. A consciencialização dos benefícios de uma boa adesão ao tratamento e o confronto com o incumprimento do jovem fictício induziu a uma reflexão e avaliação sobre o seu próprio comportamento.

Também no estudo piloto realizado por Malheiro e colaboradoras ${ }^{(13)}$, a implementação de monitores com SB peritos na autogestão "Lay Led's" foi considerada uma estratégia importante para os jovens não só pela aprendizagem mas também pela motivação, associadas à perspetiva de no futuro gerir de forma autónoma a sua condição e ainda pela possibilidade de poderem virem a ser monitores Lay Leds. Assim como, a implementação do regime de tutoria foi uma estratégia destacada por estes jovens. Consideraram o regime de tutoria como uma mais-valia, pois obrigou-os a melhorar o seu desempenho no autocuidado e na gestão da sua condição, para que o pudessem transmitir de forma correta aos mais pequenos. Apesar dos resultados reforçarem os benefícios que a utilização de modelos com a mesma condição e peritos na autogestão no âmbito do desenvolvimento de competências de autogestão nos jovens com SB, parece-nos pouco explorada pela comunidade científica.

\section{CONCLUSÃO}

O campo obteve resultados surpreendentes. Foi unânime a importância que este programa teve na capacitação para a autogestão em adolescentes com SB essenciais na conquista da autonomia do jovem com SB. Os benefícios relacionados com as estratégias de intervenção psicoeducativa utilizadas no programa (resolução de problemas, roleplaying, plano de ação e modelagem) são claramente comprovados e podemos afirmar que estão associados a uma franca melhoria nos seus comportamentos de autogestão.

As mudanças verificadas no comportamento do jovem ao assumir o papel de tutor permitem destacar a efetividade desta estratégia e o grande potencial que poderá ter na área da criança com doença crónica/incapacidade. Ressalva-se ainda que a existência de modelos de referência com SB Lay Led constituiu um fator-chave para facilitar o processo de aprendizagem, uma vez que essa pessoa, por ter vivenciado as mesmas dificuldades que estes jovens, pode fornecer as melhores estratégias para as ultrapassar. Estes resultados suportam a evidência que os programas que utilizam Lay Leds trazem benefícios para os 
jovens.

Consideramos que estes resultados podem fundamentar algumas mudanças na prática face à problemática da promoção de competências de autogestão no jovem com condição crónica e facilitação da sua transição para a vida adulta. Deste modo, pode-se considerar que a adaptação do programa de educação para a autogestão Expert Patients Program para a população pediátrica com SB foi bem conseguida e os resultados permitem afirmar que é efetivo.

Este estudo representa um importante contributo científico e inovador para a enfermagem na área do jovem com necessidades de saúde especiais no âmbito da promoção da saúde e prevenção das condições secundárias. Podemos afirmar que este programa constitui um marco importante no desenvolvimento da mestria no jovem, como facilitador de uma transição, e consequentemente, na melhoria da sua qualidade de vida relacionada com a saúde e bem-estar.

\section{REFERÊNCIAS}

1. Malheiro I. Efeito de um Programa de Educação para a Autogestão em Jovens com Spina Bífida: Desafios para a Enfermagem [tese]. Lisboa: Universidade de Lisboa - Escola Superior de Enfermagem de Lisboa; 2015. [acesso em 03 nov 2018]. Disponível em: http://hdl.handle.net/10451/20479.

2. O'Mahar K., Holmbeck GN, Jandasek B., Zukerman J. A camp-based intervention targeting independence among individuals with spina bifida. J Pediatr Psychol.[Internet]. 2010. [acesso em 05 dez 2018]; 35(8):848-856. Disponível em: http://dx.doi.org/10.1093/jpepsy/jsp125.

3. Antolovich G,Wray A. The Habilitation of Children and Young Adults with Spina Bifida. In: Özek et al (org). Spina Bifida Management and Outcome. Milan - Italy: Springer Milan - Italy: 2008. p. 341-375.

4. Peny-Dahlstrand M., Krumlinde-Sundholm L., Gosman-Hedström G. Is autonomy related to the quality of performance of everyday activities in children with spina bifida? Disabil Rehabil. [Internet]. 2012 [acesso em 06 nov 2018], 34(6):514-521. Disponível em: http://dx.doi.org/10.3109/09638288.2011.610495.

5. Soe MM, Swanson ME., Bolen JC, Thibadeau JK, Johnson N. Health risk behaviors among young adults with spina bifida. Dev Med Child Neurol [Internet]. 2012 [acesso em 21out 2018]; 201254(11):1057-1064. Disponível em: http://dx.doi.org/10.1111/j.1469-8749.2012.04402.x.

6. Sawin KJ, Bellin MH, Builta E, Vasel L, Buran CF, Brei TJ. Cross-informant agreement between adolescents with myelomeningocele and their parents. Dev Med Child Neurol [Internet].2006. [acesso em 21out 2018]; 48(3):188-194. Disponível em: http://dx.doi.org/10.1017/S0012162206000417.

7. Buran CF, Sawin KJ, Brei TJ, Fastenau PS. Adolescents with myelomeningocele: activities, beliefs, expectations, and perceptions. Dev Med Child Neurol [Internet].2004. [acesso em 24 out 2018]; 46(4):244-252. Disponível em: https://www.cambridge.org/core/services/aop-cambridge-core/ content/view/C248180E9A468ED71BBCE1AE23049D06/S0012162204000404a.pdf/adolescents-withmyelomeningocele-activities-beliefs-expectations-and-perceptions.pdf.

8. Ruck J, Dahan-Oliel, N. Adolescence and young adulthood in spina bifida: self-report on care received and readiness for the future. Top Spinal Cord Inj Rehabil [Internet]. 2010. [acesso em 20 nov 2018];16(1):26-37. Disponível em: http://dx.doi.org/10.1310/sci1601-26.

9. Sandler AD. Children with Spina Bifida: Key Clinical Issues. Pediatr. Clin. North Am [Internet]. 2010. [acesso em 20 nov 2018]; 57(4):879-892. Disponível em: https://ac.els-cdn.com/S0031395510001008/1s2.0-S0031395510001008-main.pdf? tid=9e5779da-ef6d-499d-ba3a-35404606dfe6\&acdnat $=1552913812$

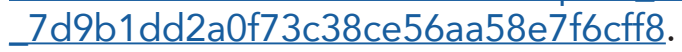

10. Holmbeck GN, Devine KA. Psychosocial and family functioning in spina bifida. Dev Disabil Res Rev [Internet]. 2010 [acesso em 25 out 2018]; 16(1):40-46. Disponível em: http://dx.doi.org/10.1002/ddrr.90. 
11. Holman $\mathrm{H}$, Lorig K. Patient self-management: a key to effectiveness and efficiency in care of chronic disease. Public Health Rep. 2004. 119(3): 239-243.

12. Malheiro MID da C; Gaspar MF, Barros L. Training Camp: Effects of an Educational Program for Selfmanagement, on Adolescents with Spina Bifida. Academy of Strategic Management Journal. Research Article. [Internet]. 2017 [acesso em 25 out 2018];16(2).Disponível em: https://www.abacademies.org/ articles/training-camp-effects-of-an-educational-program-for-selfmanagement-on-adolescents-with-spinabifida-6749.html.

13. Malheiro M, Gaspar M, Barros ML. Efeitos de um Programa de Educação para a Autogestão em Jovens com Spina Bífida: uma abordagem qualitativa. In: $6^{\circ}$ Congresso Ibero-Americano em Investigação Qualitativa: Investigação Qualitativa em Saúde; 2017 2:1175-1185; Aveiro: Ludomedia, 2017. Disponível em: https://proceedings.ciaiq.org/index.php/ciaiq2017/article/view/1454/1411.

14. Healy H, Rigby P. Promoting independence for teens and young adults with physical disabilities. Can J Occup Ther. 1999 66(5):240-249.

15. Gibbs, A. Focus Groups. Social research UPDATE, Department of Sociology, University of Surrey, United Kingdom, 1997 [acesso em 25 out 2018] 1 (19). Disponível em: http://sru.soc.surrey.ac.uk/SRU19. $\underline{\text { html. }}$

16. Dormans JP. Pediatric Ortopaedics and Sports Medicine: The Requisites in Pediatrics. D. J. P. Oxford: Mosby; 2004. p.417-436.

17. Moniz, LJ, Barros, L. Psicologia da doença para cuidados de saúde: Desenvolvimento e intervenção. Porto: ASA; 2005.

Recebido: 08/10/2018

Finalizado: 25/03/2019

Autor Correspondente:

Maria Isabel Costa Malheiro

Escola Superior de Enfermagem de Lisboa

Av. Professor Egas Moniz, 1600-190, Lisboa, Portugal

E-mail: mmalheiro@esel.pt

Contribuição dos autores:

Contribuições substanciais para a concepção ou desenho do estudo; ou a aquisição, análise ou interpretação de dados do estudo - MFG, LB

Elaboração e revisão crítica do conteúdo intelectual do estudo - MFG, LB

Aprovação da versão final do estudo a ser publicado - MFG, LB

Responsável por todos os aspectos do estudo, assegurando as questões de precisão ou integridade de qualquer parte do estudo - MICM 